\title{
Long-term persistence of response-repetition tendencies based on performance or observation
}

\author{
DAVID W. WITTER, MELVIN H. MARX, and JOHN FARBRY \\ University of Missouri, Columbia, Missouri 65201
}

\begin{abstract}
Two sessions of multiple-choice learning were administered to 172 college students. Two groups worked in pairs, one student performing (making the responses) and the other observing the performer. In the other two groups, the subjects worked individually. Each subject performed on half of the stimuli and observed on the other half, observers watching the protocol of a previous performer. The main results were that on tests given immediately after each training trial, performers reliably reported more correct and incorrect responses than observers, confirming previous results obtained under similar conditions. A secondary result was that female students showed reliably greater repetition of incorrect responses under social conditions.
\end{abstract}

Previous studies from this laboratory (Marx \& Witter, 1972; Marx, Witter, \& Farbry, 1973) indicate that subjects working on a multiple-choice learning task repeat incorrect as well as correct responses more often after performing (subject makes own response) than after observing (subject watches the response of another subject). The present experiment examined response repetition and number of correct responses as a function of performance/observation, method of observation (direct vs. indirect), and sex, in two sessions, 1 month apart, in order to determine the extent to which response-repetition tendencies persist over a long retention interval.

\begin{abstract}
Subjects and Design
Two groups of females and two groups of males, totaling 172 students from an introductory psychology course, served as subjects in the experiment. Each group was tested in two sessions, 1 month apart. The within-sex groups were differentiated by method of observation. In the direct observation (social) condition, one group of each sex was randomly divided into pairs which shared the experimental materials; observers were thus instructed to observe and remember the responses of their partners. In the indirect observation (individual) condition, the other groups were treated similarly except that the observers were given written response protocols of subjects tested previously on the same stimuli.
\end{abstract}

\section{Apparatus}

The stimuli were 40 slides of hand-drawn facial features; eyes, ears, nose, or hair were the features depicted in black

This research was supported in part by Research Career Award 1-K6-MH-22-023 from the National Institute of Mental Health and by Grant DAHC19-74-G-0008 from the U.S. Army Research Institute for the Behavioral and Social Sciences, to the second author. The opinions stated in this report are those of the authors and are in no way to be considered as endorsed by the U.S. Army. David W. Witter's present address is: U.S. Army Research Institute for the Behavioral and Social Sciences, Arlington, Virginia. and white line drawings. In the first session, color slides of scenery were used as a sample set.

Subjects were required to associate a predetermined response (A, B, C, or D) with each of the 40 stimuli by guessing in a trial-and-error procedure. Subjects performed on a Rapid Rater, which provides immediate knowledge of results; indication of an incorrect response did not specify the correct response. (See Marx \& Witter, 1972, for a detailed description of this device.)

\section{Procedure}

On each of five trials in Session 1 and four trials in Session 2, each subject performed on one set of 20 slides with the Rapid Rater and observed the responses of another subject for the other set of 20 slides. Following this training, all subjects completed the trial with a pencil and paper test (with no feedback) on all 40 slides. Because slide sequences were completely randomized between trials, subjects sometimes performed on stimuli which they had previously observed, and vice versa. Presentation time was $15 \mathrm{sec}$ per slide for training, during which subjects viewed the slides and responded on the Rapid Rater; $8 \mathrm{sec}$ per slide was given for the test portion of a trial. Time between trials was approximately $1 \mathrm{~min}$, and the interval between training and test portions of a trial was approximately $30 \mathrm{sec}$.

The same procedure was used in both sessions except that no sample slides were shown and only four training trials were given in Session 2. Session 2 began with a test trial to assess retention over the intervening 1 -month period, making a total of five test trials for that session. Thus, the factorial design consisted of two between-group variables (method of observation and sex) and two within-group variables (perform/observe and trials) for each session.

\section{RESULTS}

Three measures of performance were taken: incorrect response repetition (IRR), correct response repetition (CRR), and number of correct responses (NCR). Response repetition was defined as making the same response to a stimulus on both training and test portions on any given trial. An arc sine transformation was applied to the responserepetition data. 


\section{Correct Response Repetition and Incorrect \\ Response Repetition}

In Session 1, as shown in Figure 1, performers demonstrated both higher CRR, $F(1,84)=10.71$, $\mathrm{p}<.005$, and higher IRR, $\mathrm{F}(1,84)=28.01$, $\mathrm{p}<.001$. The CRR measure increased across trials, $\mathrm{F}(4,336)=35.49, \mathrm{p}<.001$, but there was no trials effect for IRR, $F(4,336)=2.07, p>.05$. An interaction between Perform/Observe and Trials occurred in Session 1 for both CRR, $F(4,336)=$ $3.40, \mathrm{p}<.025$, and IRR, F(4,336) = 3.05, p $<.025$. These interactions occurred primarily between Trial 1, where there was little or no difference between performers and observers, and Trial 2, where performers showed higher response repetition which was maintained on subsequent trials. Except for this interaction, these results closely duplicate the results of Marx and Witter (1972) and partially duplicate the results of Marx et al. (1973); in the latter study, there was no Trials effect for CRR in the first two of the three sessions.

In Session 2, these main effects were repeated. Scores again increased with trials for CRR, $F(3,204)$ $=31.22, p<.001$. Performers produced more CRR, $F(1,68)=21.68, p<.001$, and more IRR, $\mathrm{F}(1,68)=33.56, \mathrm{p}<.001$, than observers. Females showed superiority over males in CRR, $F(1,68)=$ $5.59, \mathrm{p}<.025$.

\section{Number of Correct Responses}

The NCR measure revealed the expected reliable main effect of trials in both Sessions 1 and 2, $\mathrm{F}(4,336)=37.44, \mathrm{p}<.001$, and $\mathrm{F}(4,272)=53.29$, $\mathrm{p}<.001$, respectively. No reliable difference was found for the Perform/Observe variable. A more analytic measure of NCR, using only those stimuli which were consistently either performed or observed by an individual subject, resulted in two additional reliable main effects for Session 2: Sex, with females superior, $F(1,68)=5.09, \mathrm{p}<.05$, and Condition, with performers superior, $\mathrm{F}(1,68)=5.34, \mathrm{p}<.025$. The direction of the differences matches the corresponding main effects on CRR in Session 2.

\section{Interaction of Method of Observation and Sex}

In Session 1, the IRR measure revealed an interaction of Sex with Method of Observation, $F(1,84)=4.02, p<.05$. Females showed less IRR (i.e., superior performance) in the individual condition than in the social condition, whereas males showed slightly more IRR in the individual condition than in the social condition.

The interaction of Sex with Method of Observation for IRR did not occur in the second session, nor was method of observation involved in any other major effects. This fact suggests that the apparent dependence of female responding on type of observation method dissipates with further practice.

\section{DISCUSSION}

\section{Response-Repetition Tendencies}

In this experiment, a given stimulus was sometimes used in the performance condition, and at other times used in the observation condition, for an individual subject. In spite of this more rigorous test of the differential repetition tendency for performers and observers, the results closely duplicated those of previous experiments in which the more typical arrangement of consistent assignment of stimuli either to the performer or the observer condition obtained. This fact suggests that there is a substantial contribution of intratrial factors to the response-repetition tendency. In other words, the probability of a particular response being associated with a given stimulus seems largely to depend upon whether that stimulus is presented in the performance or the observation condition. During performance, the probability of forming and maintaining both correct and incorrect associations is higher than during observation.

The Trials by Performance/Observation interaction for CRR in Session 1, which was not matched in Session 2, suggests that the greater tendency to repeat correct responses under performance conditions developed over trials in Session 1 and

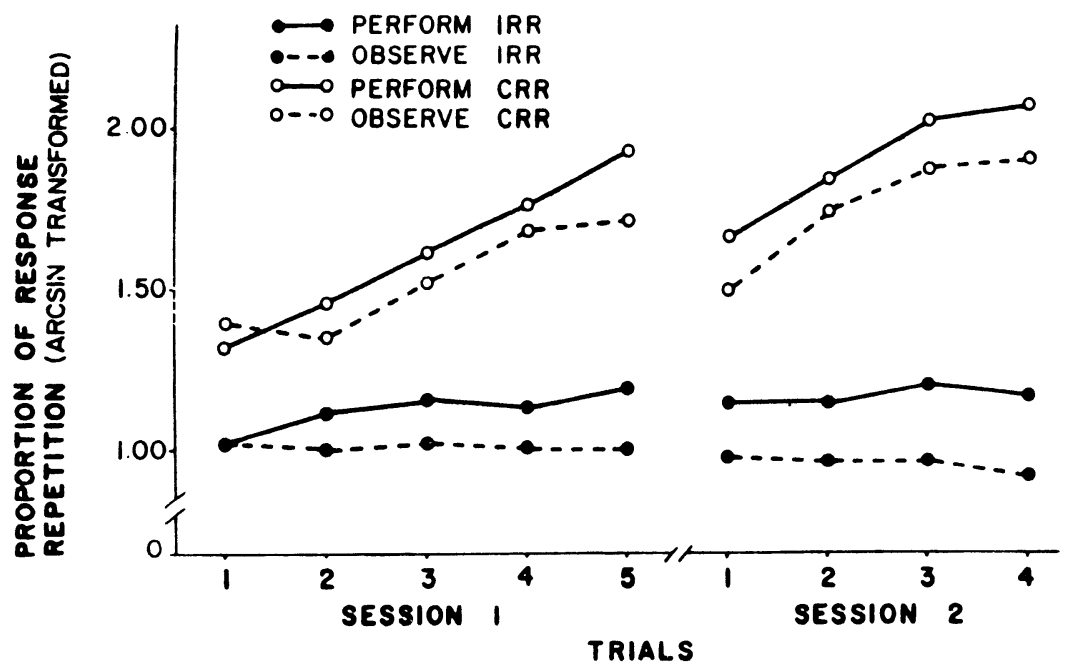

Figure 1. Correct response repetition (CRR) and incorrect response repetition (IRR) as a function of performance and observation in two sessions. 
persisted over the intersession interval; performers showed reliably more repeating of correct responses in both sessions. Similarly, for errors, the Trials by Performance/Observation interaction was reliable in Session 1 only, while performers showed reliably more IRR in both of the sessions. These results thus give further support to the hypothesis that these response-repetition tendencies are a function of performing a task, in that they increase more markedly for performers. Nevertheless, the two repetition tendencies (for errors as well as correct responses) may cancel out and so yield no difference in acquisition, as measured in number of correct responses, between performance and observation conditions.

The most notable new finding in these data is that an interval as long as 1 month can interrupt the acquisition process and yet allow the greater response-repetition tendency generated under performance conditions to persist. This finding is especially significant in that the measures of CRR and IRR were not taken for consistently performed or observed stimulusresponse units, as mentioned above. The response-repetition effects here demonstrated have three important features: (1) they develop over trials (as shown by the reliable interaction of Performance/Observation and Trials in Session 1), and thus cannot be explained merely on the basis of differential pretraining response strengths; (2) once developed, they are robust over time; (3) they seem to be confined primarily to short-term retention, i.e., they are more specific to the immediate (intratrial) status of each stimulus than to its accumulated "history" of performance vs. observation.

This lack of stimulus specificity is further evidence for the acquired habitual nature of the response repetition, as earlier discussed (Marx \& Witter, 1972). However, other recent evidence from this laboratory (Marx. 1971; Marx. Witter. \& Mueller. 1972a) suggests that the probability of an error being repeated also depends upon how many times that same response has been made, incorrectly, on previous trials; the more often the response has been incorrect, on successive trials, the more likely it is to be made on the following trial. Repetition of errors thus appears to be affected by both within-trial and between-trial processes. More analytic data than are ordinarily reported (e.g., as in the number of correct responses) will be required to separate the relative influences of these two basic factors.

\section{Sex Difference}

The pattern of the Sex by Method of Observation interaction is similar in this experiment to the Sex by Method of Observation by Trials interaction earlier reported by Marx, Witter, and Mueller (1972b). In that study, female subjects also showed reliably inferior performance in the social conditions, as measured by number of correct responses. The failure of a difference in overall learning rate (number of correct responses) to occur in this study, whereas the more sensitive IRR measure did show an inferiority for females in the social condition, may reflect the lesser degree of variation between social and individual conditions in this experiment (subjects sitting next to each other, while in the older experiment they were physically isolated, in separate booths).

\section{REFERENCES}

MARX, M. H. Increased probability of error repetition as a function of number of successive prior repetitions. Perceptual and Motor Skills, 1971, 32, 544-546.

MARX, M. H., \& WitTER, D. W. Repetition of correct responses and errors as a function of performance with reward or information. Journal of Experimental Psychology, 1972, 92, 53-58.

MarX, M. H., Witter, D. W., \& Farbry, J. Greater repetition of errors under performance compared to observation in multiple-choice human learning. Perceptual and Motor Skills, 1973, 37, 949-950.

MarX, M. H., Witter, D. W., \& Mueller, J. H. Additional data on probability of error repetition following varying numbers of successive prior repetitions. Perceptual and Motor Skills, 1972, 34, 525-526. (a)

MARX, M. H., WitTer, D. W.. \& MUelleR, J. H. Interaction of sex and training method in human multiple-choice learning. Journal of Social Psychology, 1972, 88, 37-42. (b)

(Received for publication March 9, 1976.) 